\title{
Simulation Analysis on Optimal Guidance Law of Maneuvering Target Based on Multiple Model Adaptive Estimator
}

\author{
Shengnan $\mathrm{Fu}^{1, a}$, Shixiang Liu ${ }^{1, b}$ and Qunli Xia ${ }^{1, b}$ \\ ${ }^{1}$ School of Aerospace Engineering, Beijing Institute of Technology, Beijing 100081,China \\ aqcqgfmn@163.com, ${ }^{b} 1010 @$ bit.edu.cn
}

\begin{abstract}
Keywords: Kalman filter;Multiple model adaptive Estimator(MMAE);Optimal guidance law;Sinemaneuvering;Matlab simulation.
\end{abstract}

\begin{abstract}
Aiming at randomly sine-maneuvering target tracking in the precision guided system, a fast and efficient algorithm is proposed for multiple model adaptive estimation. The filter bank corresponding to target maneuvering model was adopted to estimate the acceleration of the target, which can improve the estimated accuracy of the acceleration of the target. At the same time, the optimal guidance law based on sine-maneuvering target is adopted for the weave maneuver, in order to ensure the guidance law, the filter and the target maneuvering model remain in a consistent state, which can improve the guidance accuracy of the maneuvering target. By analyzing the simulation result, it shows that this method has good tracking performance, and multiple model adaptive filtering can be widely used in tracking in the precision guided system.
\end{abstract}

\section{Introduction}

In the modern war, the guided interceptor must possess precise guidance and even hit the target directly. Proportional navigation is the optimal guidance law to solve the guidance problem without time-lag and maneuvering targets. With a maneuvering target and time- lag, proportional navigation is no longer the optimal guidance law. The mismatching problems between filter model and the real target motion model can lead to low filtering accuracy, which makes the guidance system lose its accuracy.

This paper obtains the weave guidance law[1] based on weave maneuvering targets and time-lag of the guidance system, which is the optimal guidance law in the sense. If the weave frequency of the target is known in advance, a four-state linear weave Kalman filter can be designed to yield excellent estimates of the necessary states to implement the weave guidance law. When the target weaving frequency is unknown, the filter model based on multiple model adaptive estimator (MMAE) has been established. An effective method is presented to perform the target tracking when the weave frequency is unknown. At last, through the analysis of simulation results compared with extended Kalman filter (EKF) algorithm, the efficiency of the proposed algorithm has been proved.

Description of the Problem.The intercept problem of homing missile in three-dimensional space is simplified to two-dimensional plane in this paper[2]. The description of the intercept problem in the sight coordinate is shown in Fig. 1.X-axis was used as initial sight, and subscript $\mathrm{M}$ and subscript $\mathrm{T}$ correspond to associated states of the interception missile and the target respectively. $\mathrm{R}$ is the relative distance between the missile and the target, and $\mathrm{y}$ is the initial relative displacement. It assumes that the target is implementing sine-wave maneuver and the maneuvering frequency is known. There is one time constant in the intercept missile’s guidance loop. 


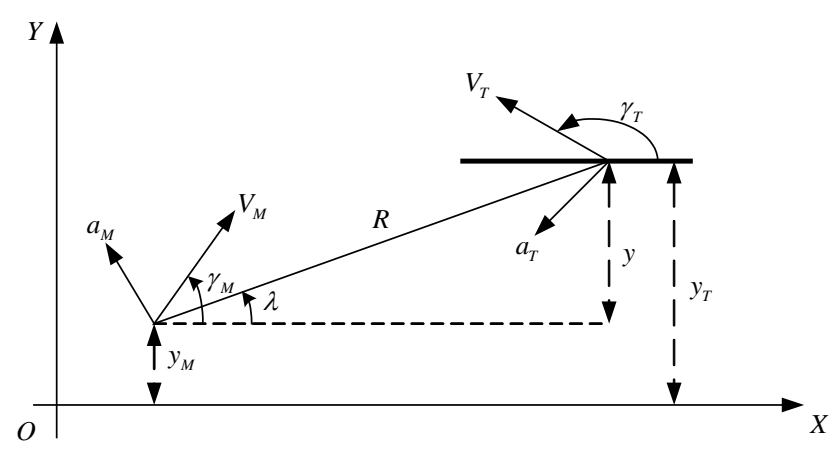

Fig. 1. Relative motion model in two-dimensional plane

The four-state space model for the missile against a sine-weave target is established and shown $\mathrm{i}$ $\mathrm{n}$ Eq.1, where $y$ represents relative position; $\dot{y}$ represents relative velocity; $\ddot{y}$ represents target acceler ation; $\dddot{y}$ represents target jerk[3,4].

$$
\left[\begin{array}{c}
\dot{y} \\
\ddot{y} \\
\dddot{y}_{T} \\
\dddot{y}_{T}
\end{array}\right]=\left(\begin{array}{cccc}
0 & 1 & 0 & 0 \\
0 & 0 & 1 & 0 \\
0 & 0 & 0 & 1 \\
0 & 0 & -\omega^{2} & 0
\end{array}\right)\left[\begin{array}{c}
y \\
\dot{y} \\
\ddot{y}_{T} \\
\dddot{y}_{T}
\end{array}\right]+\left[\begin{array}{c}
0 \\
-\frac{1}{\tau} \\
0 \\
0
\end{array}\right] n_{c}
$$

In the formula, $\tau$ represents the intercept missile's guidance system time constant; $n_{c}$ represents the intercept missile's acceleration command and $\omega$ represents the weave frequency of the target.

Generally, the distance $R$ and the line-of-sight angle $\lambda$ between the missile and the target can be measured by a radar seeker. It assumes that $R$ can be measured precisely and $\lambda$ has the limited noise. The noise is white noise which follows the Gaussian distribution and the standard variance is $\sigma_{\lambda}$. The measurement equation is shown in Eq.2.

$$
z(\mathrm{t})=[1 / \mathrm{R} \quad 0 \quad 0 \quad 0] \mathrm{y}(\mathrm{t})+v(\mathrm{t})
$$

Guidance Law. The weave guidance law is optimal in the sense that it requires the least acceleratio $\mathrm{n}$ against weave maneuvers[5]. The weave guidance law is shown to be

$$
n_{c}=\frac{N^{\prime}}{t_{g o}^{2}}\left[y+\dot{y} t_{g o}+\left(\frac{1-\cos \omega t_{g o}}{\omega^{2}}\right) \ddot{y}_{T}+\left(\frac{\omega t_{g o}-\sin \omega t_{g o}}{\omega^{3}}\right) \dddot{y}_{T}-n_{L} \tau^{2}\left(e^{-x}+x-1\right)\right]
$$

where $x$ is given by $x=t_{g o} / \tau$ with $t_{g o}$ being the time to go until intercept. The effective navigation ratio is given by

$$
N^{\prime}=\frac{6 x^{2}\left(e^{x}-1+x\right)}{2 x^{3}+3+6 x-6 x^{2}-12 x e^{-x}-3 e^{-2 x}}
$$

This weave guidance law makes use of four state estimates from the filter (that is, estimates of relative position, relative velocity, target acceleration, and target jerk). Essentially the weave guidance law makes an internal prediction of where the target will be at intercept. This information enables the missile to guide to the predicted intercept point using a minimum of energy.

The proportional navigation (PN) and the optimal proportional navigation (OPN) are also presented in Eq.4 and Eq.5, in order to compare the performance of guidance with weave guidance law.

$$
n_{P N}=\frac{N^{\prime}}{t_{g o}^{2}}\left[y+\dot{y} t_{g o}\right]
$$




$$
n_{O P N}=\frac{N^{\prime}}{t_{g o}^{2}}\left[y+\dot{y} t_{g o}+\frac{t_{g o}^{2}}{2} \ddot{y}_{T}-n_{L} \tau^{2}\left(e^{-x}+x-1\right)\right]
$$

MMAE Algorithm. The idea of multiple model algorithm was proposed in 1985 by Magill, which has been extensively applied to state estimation problems for hybrid systems. In this technique several filters are run in parallel with each one being tuned to a different parameter value[6,7]. The likelihood function and Bayes' rule are used to determine the probability that a filter is the correct one. Estimates from each of the filters are weighted by the probability and combined to form a resultant estimate.

The MMAE is built from $\mathrm{N}$ elemental filters. Processing the measurement at time $t_{k}$ by all filters yields $\mathrm{N}$ versions of the innovation process at $t_{k}$ :

$$
r_{j}(k)=z(k)-H_{j}(k) \hat{x}_{j}(k \mid k-1), j=1,2, \ldots, N
$$

Where $z(k), H_{j}(k)$ and $\hat{x}_{j}(k \mid k-1)$ are the measurement vector, the observation matrix and the priori state estimate of the jth elemental filter at time $t_{k}$. With the innovation process of each filter, the posteriori probability that its design hypothesis is true can be recursively computed as:

$$
p_{j}(k)=\frac{f_{j}(k) p_{j}(k-1)}{\sum_{i=1}^{N} f_{i}(k)}
$$

Where the probability density function $f_{j}(k)$ is

$$
f_{j}(k)=\left\{1 /(2 \pi)^{m / 2}\left[\operatorname{det} \tilde{P}_{j}(k)\right]^{\frac{1}{2}}\right\} \exp \left\{-\frac{1}{2} r_{j}^{T}(k) \tilde{P}_{j}^{-1}(k) r_{j}(k)\right\}
$$

The innovation process covariance is

$$
\tilde{P}_{j}^{-1}(k)=H_{j}(k) P_{j}(k \mid k-1) H_{j}^{T}(k)+R_{j}(k)
$$

and $P_{j}(k \mid k-1)$ and $R_{j}(k)$ are the a priori estimation error covariance and the measurement noise covariance at time $t_{k}$,respectively. After all posteriori probabilities have been computed, The MMAE estimate can be computed as a probability weighted sum of all elemental state estimates.

Simulations and Results. The miss distance analysis of the weaving target problem is presented for the guidance system of Fig. 2. The filter, shown in the homing loop of Fig. 2, can either be a MMAE or an extended Kalman filter. The guidance laws are weave guidance law, PN and OPN. 


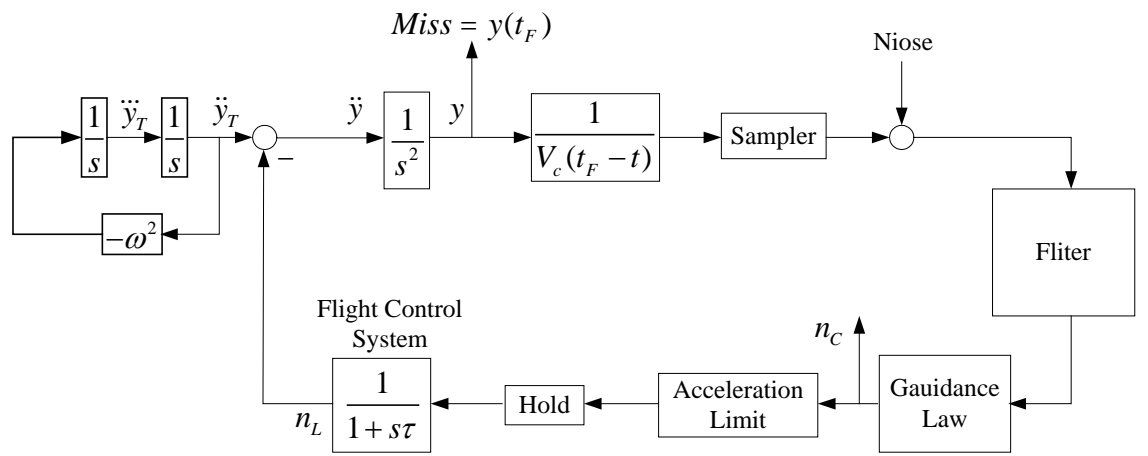

Fig. 2. Guidance system model for miss distance analysis

The parameters to be used in the miss distance analysis are listed in Table. 1. We can see from the table that the weaving target has a 3 g maneuver amplitude and a weave frequency of $2 \mathrm{r} / \mathrm{s}$. The missile has a limited acceleration for $10 \mathrm{~g}$. The flight control system time constant is set at $.5 \mathrm{~s}$. There are three linear weave Kalman filers in MMAE, which frequencies are 1rad/s, 2rad/s and 3rad/s respectively. The sample time is $0.01 \mathrm{~s}$ and the flight time varies for 0 to $10 \mathrm{~s}$. The noise in the seeker is white noise and the variance is $0.1 \mathrm{mrad}$ or $1 \mathrm{mrad}$.

Table. 1. Simulation parameters

\begin{tabular}{cccc}
\hline parameter & values & parameter & values \\
\hline$n_{T}$ & $3 g$ & $w_{1}$ & $1 \mathrm{r} / \mathrm{s}$ \\
$n_{L I M}$ & $10 \mathrm{~g}$ & $w_{2}$ & $2 \mathrm{r} / \mathrm{s}$ \\
$V_{c}$ & $3000 \mathrm{~m} / \mathrm{s}$ & $w_{3}$ & $3 \mathrm{r} / \mathrm{s}$ \\
$\tau$ & 0.5 & $T_{s}$ & $0.01 \mathrm{~s}$ \\
$w$ & $2 r / s$ & $T_{F}$ & $0 \sim 10 \mathrm{~s}$
\end{tabular}

Experiments were run with the parameters in different conditions. The results are shown in Fig.3 Fig. 6 the when the noise is $0.1 \mathrm{mrad}$. The posteriori probabilities of elemental filters are shown in Fig. 3. The MMAE takes about 3s to identify the hypothetical model which is the closest to being true. Estimation of maneuvering frequency is shown in Fig. 4.The MMAE gets an accurate frequency after 3s while the EKF takes more time to get a less accuracy answer.

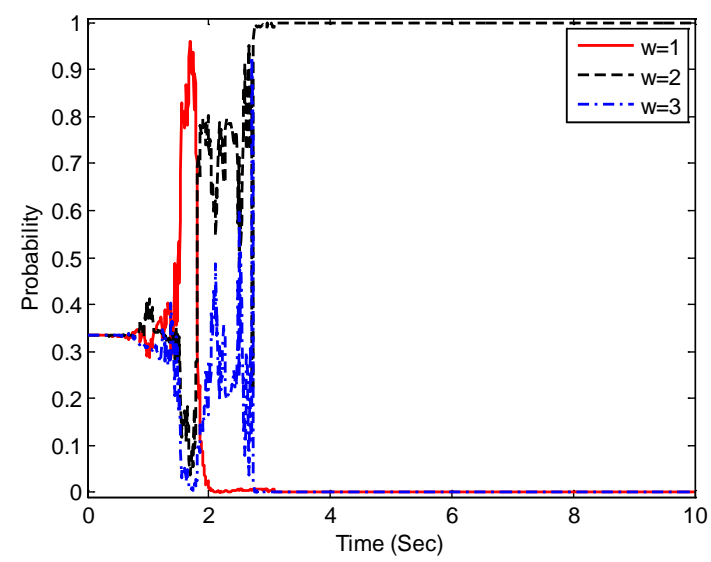

Fig. 3. Posteriori probability of elemental filters

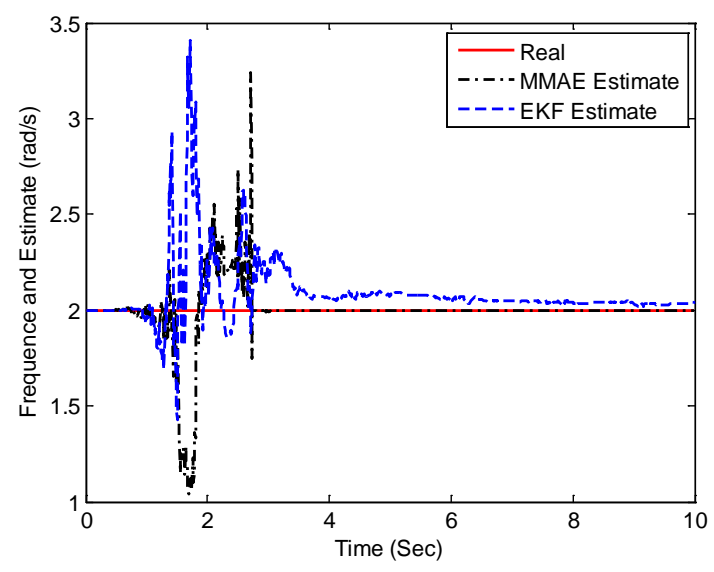

Fig. 4. Estimation of maneuvering frequency

Estimation performance of target acceleration is shown in Fig. 5.And estimation performance of target jerk is shown in Fig. 6.i.It can be seen that the MMAE filter has good tracking performance of weaving targets. 


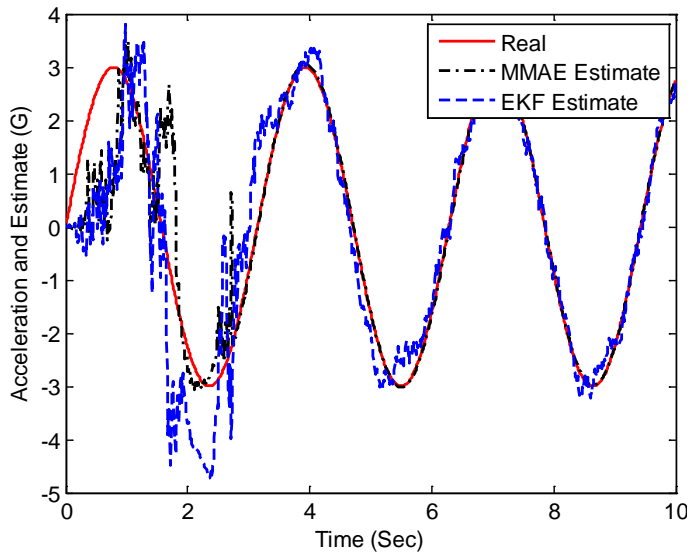

Fig. 5. Estimation of target acceleration

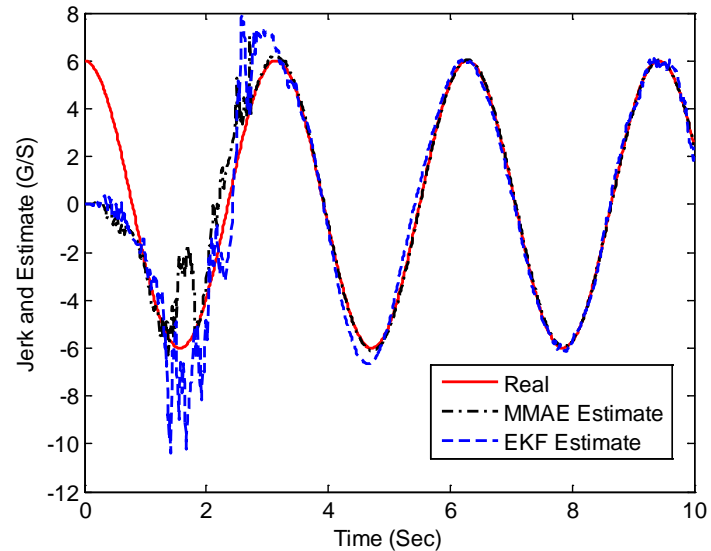

Fig. 6. Estimation of target jerk

Another experiment was run when the noise is increased from $0.1 \mathrm{mrad}$ to $1 \mathrm{mrad}$. The results are shown in Fig.7 Fig.10. We can see that MMAE takes more time to identify the correct frequency with lager noise from Fig.7 and Fig.8, while the performance of EKF decreases and take more noise in the estimate of frequency.Fig.9 and Fig.10 show that MMAE has a good performance in estimation target acceleration and target jerk than EKF.

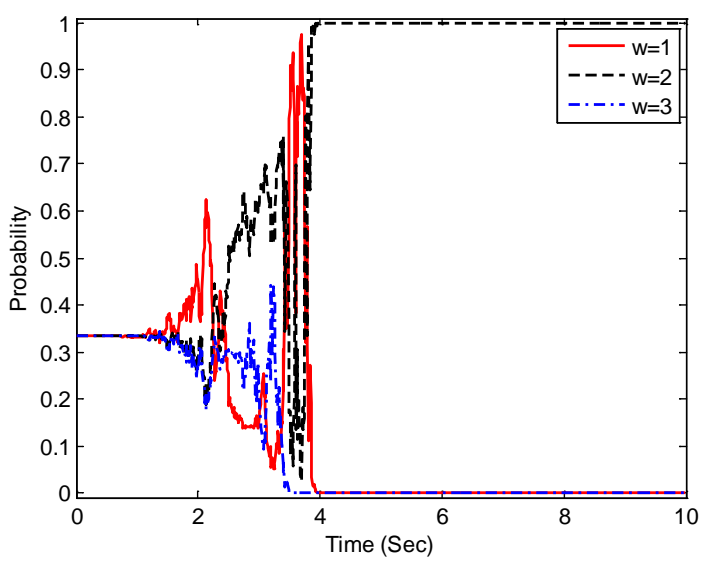

Fig. 7. Posteriori probability of elemental filters

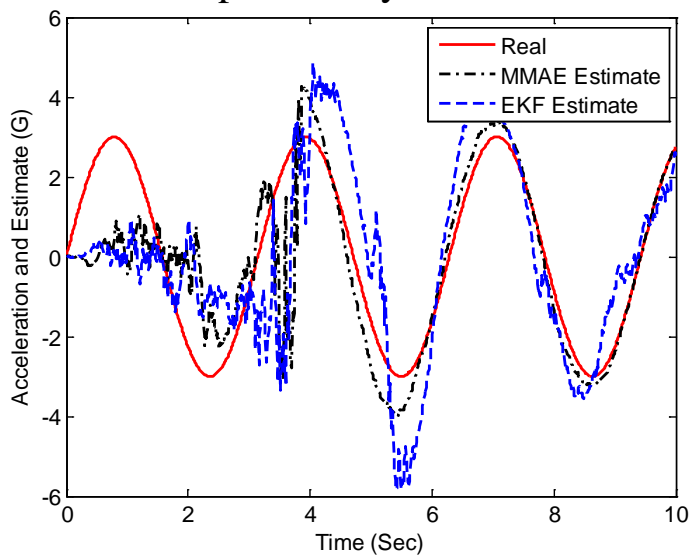

Fig. 9. Estimation of target acceleration

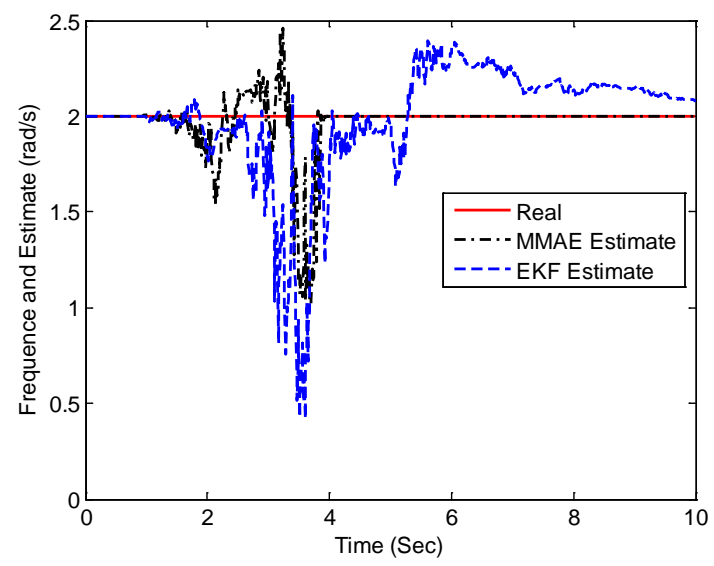

Fig. 8. Estimation of maneuvering frequency

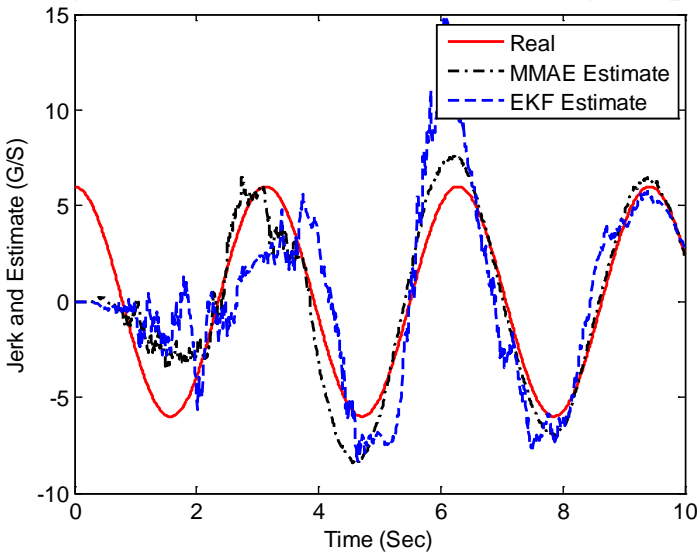

Fig. 10. Estimation of target jerk

It is obviously that MMAE has a better performance than EKF when the true frequency is matched with a filter in MMAE. We will take MMAE to analysis the performance of weave guidance law. One hundred Monte Carlo sets were run for flight times ranging from $1 \mathrm{~s}$ to $10 \mathrm{~s}$ in steps of $0.1 \mathrm{~s}$ for different noises. The results are shown in Fig.11 and Fig.12. 


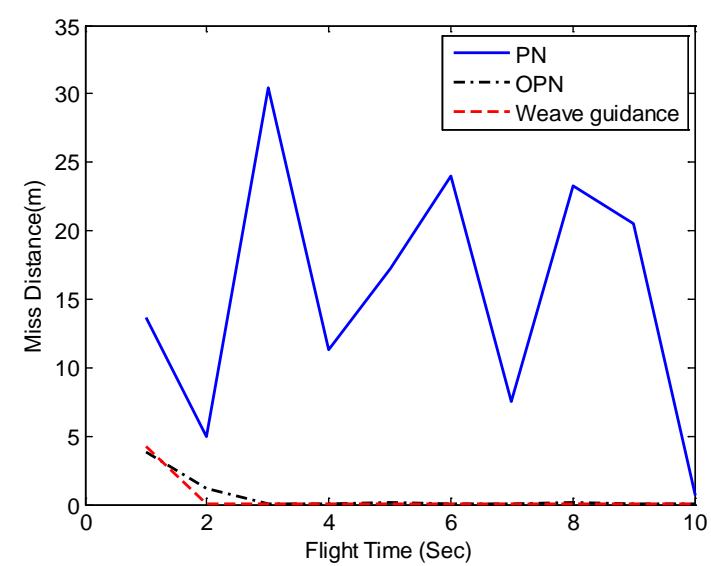

Fig. 11. Miss distance with 0.1mrad

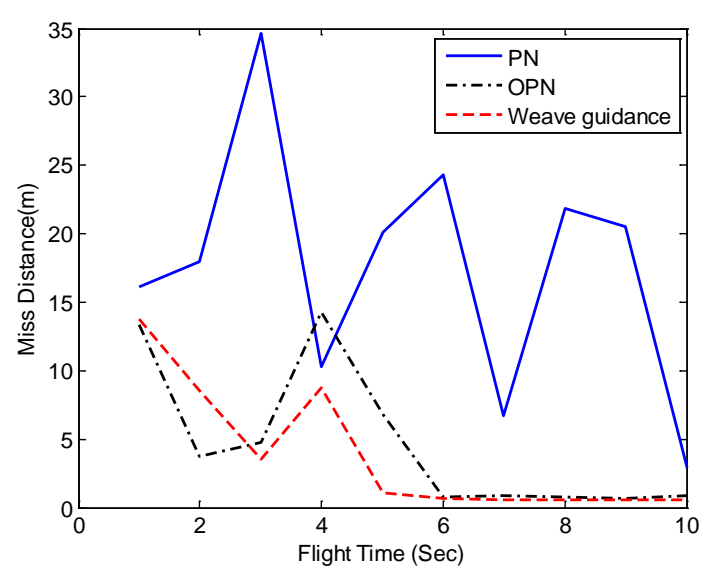

Fig. 12. Miss distance with1mrad

Fig.11 shows that both weave guidance and OPN have a good performance than PN with a low noise. In fact weave guidance gets the best performance in the three guidance laws. Fig.12 shows that miss distance increase for three guidance law with a large noise. The performance of PN seems to change little. The performance of weave guidance is less better than OPN for a short flight time because of more noise included in the filter. For a large flight time the weave guidance law can get the lowest miss distance.

\section{Conclusions}

From the simulation results we can see that if the maneuvering frequency of the target is unknown, by using MMAE algorithm we can get a better filter performance than EKF. MMAE takes 3 4 s to get the correct frequency and have a good estimate of target acceleration and jerk. For a limited missile acceleration, the weave guidance law matches to target maneuver using MMAE has the least miss distance with low noise. For a long flight time it also has the best guidance performance.

\section{References}

[1] Zarchan, P.,Tactical and Strategic Missile Guidance,3rd ed.,AIAA,VA,pp.585-595.1998

[2] X.R. Li and V.P. Jilkov:A survey of maneuvering target tracking-part III:Measurement models, Proc.2001 SPIE Conf.Signal and Data Processing of Small Targets,San Diego,CA,vol. 4473,pp.423-446,2001.

[3] X.R. Li,Y.Zhu,J.Wang,and C.Han:Optimal linear estimation fusion-part I: unified fusion rules, IEEE Transactions on Information Theory,vol.49,no.9,pp.2192-2208,2003.

[4] K.V. Ramachandra:Optimum steady state position,velocity,and acceleration estimation using noisy sampled position data,IEEE Trans.on Aerospace and Electronic Systems,vol.23,pp.705708,1987.

[5] M.E. Liggins II,C.Y. Chong,I.Kadar,M.G. Alford,V.Vannicola,and S.Thomopoulos:Distributed fusion architectures and algorithms for target tracking, Proc.of The IEEE,vol.85,no.1,pp.95-107, 1997.

[6] P.S. Maybeck and R.D. Stevens:Reconfigurable flight control via multiple model adaptive control methods,IEEE Transactions on Aerospace and Electronic Systems,27,3,pp.470-480.1991

[7] X.R. Li and Y.Bar-Shalom:Multiple model estimation with variable structure,IEEE Transactions on Automatic Control,41,4,pp.479-493.1996 